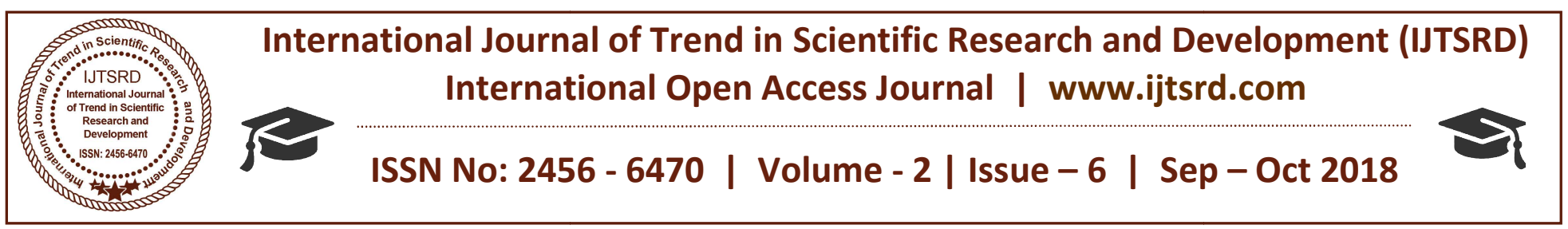

\title{
Identifying the Slider-Crank Mechanism System by the MPSO Method
}

\author{
Chin Wen Chuang, Kuan Yu Chen, Yi Hua Su \\ Department of Electrical Engineering, I-Shou University \\ Kaohsiung, Taiwan
}

\begin{abstract}
This paper mainly proposes an efficient modified particle swarm optimization (MPSO) method, to identify a slider-crank mechanism driven by a fieldoriented PM synchronous motor, dynamic formulations of a slider-crank mechanism have been successfully formulated with only one independent variable. The parameters of many industrial machines are difficult to obtain if these machines cannot be taken apart. In system identification, we adopt the MPSO method to find parameters of the slider-crank mechanism. This new algorithm is added with "distance" term in the traditional PSO's fitness function to avoid converging to a local optimum. It is found that the MPSO method can obtain optimal highquality solution, high calculation efficiency, and its feasibility and effectiveness. Finally, the comparisons of numerical simulations and experimental results prove that the MPSO identification method for the slider-crank mechanism is feasible.
\end{abstract}

Keyword: Slider-crank mechanism, Modified particle searm optimization.

\section{INTRODUCTION}

A slider-crank mechanism is widely used in gasoline and diesel engines, and has been studied extensively in the past three decades. The responses of the system found by Viscomi and Ayre [1] are to be dependent upon the five parameters as the length, mass, damping, external piston force and frequency. The steady-state responses of the flexible connecting rod of a slider-crank mechanism with time-dependent boundary effect were obtained by Fung [2]. A slidercrank mechanism with constantly rotating speed was controlled by Fung et al. [3]. The mathematical model of the coupled mechanism of a slider-crank mechanism was obtained by Lin et al. [4], where the system is actuated by a field-oriented control permanent magnet (PM) synchronous servomotor.
However, the dynamic formulations of a slider-crank mechanism with one degree-of -freedom have more than one independent variable in the past researches [3-4]. In this study, the dynamic formulation is expressed by only one independent variable of rotation angle. Moreover, its dynamic responses are compared well with the experimental results.

Particle swarm optimization (PSO) is a stochastic population based optimization approach, and was first published by Kennedy and Eberhart in 1995 [5-6] PSO has been shown to be an efficient, roust and simple optimization algorithm. Most studies of the PSO are empirical with only a few theoretical analyses that concentrate on understanding particle trajectories. A large amount of research has been proposed to improve the performance of PSO. From these studies, much effort has been invested to obtain a better understanding of its convergence properties. These -studies concentrated mostly on a better understanding of the basic PSO control parameters, namely the acceleration coefficients, inertia weight, velocity clamping, and swarm size [7-9]. In this paper, a modified PSO algorithm is proposed to improve the searching ability and prevent from being trapped in a local optimum. The main difference of the MPSO from the PSO is its fitness function considers the "distance" to avoid converging to a local optimum. From these empirical studies it can be concluded that the MPSO is sensitive to control parameter choices, specifically the inertia weight, acceleration coefficients and velocity clamping. However, wrong initialization of these parameters may lead to divergent or cyclic behavior.

This study successfully demonstrates that the dynamic formulation can give a wonderful interpretation of a slider-crank mechanism in comparison with the experimental results. Furthermore, a new identified method using the MPSO is proposed, and it is 
confirmed that the method can perfectly searches the parameters of the slider-crank mechanism driven by a servomotor through the numerical simulations and experimental results.

\section{DYNAMICS FORMULATION OF A SLIDER-CRANK MECHANISM}

A slider-crank mechanism is a single looped mechanism with a very simple construction shown in Fig.1(a) and the experimental equipment of a slidercrank mechanism is shown in Fig. 1(b). It consists of three parts: a rigid disk, which is driven by a servomotor, a connecting rod and a slider.

Figure1 (a) shows the physical model of a slider-crank mechanism, where the mass center and the radius of the rigid diskare denoted as point " $O$ " and length " $r$ ", respectively. And " $l$ " is denoted as the length of the connected $\operatorname{rod} A B$. The angle $\theta$ is between $O A$ and the $\mathrm{X}$-axis, while the angle $\phi$ is between the rod $A B$ and the $\mathrm{X}$-axis. In $\mathrm{OXY}$ plane, the geometric positions of gravity centers of rigid disk, connected rod, and slider, respectively, are as follows:

$$
\begin{aligned}
& x_{1 c g}=0, \quad y_{1 c g}=0 \\
& x_{2 c g}=r \cos \theta+\frac{1}{2} l \cos \phi, y_{2 c g}=\frac{1}{2} l \sin \phi \\
& x_{3 c g}=r \cos \theta+l \cos \phi, \quad y_{3 c g}=0 .
\end{aligned}
$$

The mechanism has a constrained condition as follows

$r \sin \theta=l \sin \phi$.

The angle $\phi$ can be found from Eq. (4) as

$\phi=\sin ^{-1}\left(\frac{r}{l} \sin \theta\right)$.

In the kinematic analysis, taking the first and second derivates of the displacement of slider $B$ with respect to time, the speed and acceleration of slider $B$ are as follows

$$
\begin{aligned}
& \&=-r \& \sin \theta-l \phi \sin \phi \\
& =-r \& \sin \theta-r \Leftrightarrow \cos \theta-l \phi \sin \phi-l \phi \cos \phi .
\end{aligned}
$$

Similarly, the angular velocity $\phi^{\&}$ and acceleration are obtained as follows

$$
\begin{aligned}
& \phi=\frac{r \Leftrightarrow \cos \theta}{l \cos \phi} \\
& \frac{r \cos \phi \cos \theta+r \theta \cos \theta \sin \phi-r \theta^{2} \sin \theta \cos \phi}{l \cos ^{2} \phi} .
\end{aligned}
$$

A machine model of a PM synchronous motor can be described in a rotor rotating by [10] as follows:

$$
\begin{aligned}
& v_{q}=R_{s} i_{q}+p \lambda_{q}+w_{s} \lambda_{d} \\
& v_{d}=R_{s} i_{d}+p \lambda_{d}-w_{s} \lambda_{q}
\end{aligned}
$$

\section{Where}

$\lambda_{q}=L_{q} i_{q}$,

$\lambda_{d}=L_{d} i_{d}+L_{m d} I_{f d}$.

In the above equations, $v_{d}$ and $v_{q}$ are the $d$ and $q$ axis stator voltages, $i_{d}$ and $i_{q}$ are the $d$ and $q$ axis stator currents, $L_{d}$ and $L_{q}$ are the $d$ and $q$ axis inductances, $\lambda_{d}$ and $\lambda_{q}$ are the $d$ and $q$ axis stator flux linkages, and $R_{s}$ and $w_{s}$ are the stator resistance and inverter frequency, respectively. In Eq. (13), $I_{f d}$ is the equivalent $d$-axis magnetizing current, and $L_{m d}$ is the $d$-axis mutual inductance. The electric torque is

$\tau_{m}=\frac{3}{2} p\left[L_{m d} I_{f d} i_{q}+\left(L_{d}-L_{q}\right) i_{d} i_{q}\right]$

and the equation for the motor dynamics is

$\tau_{e}=\tau_{m}+B_{m} \omega_{r}+J_{m} \&$.

In Eq (14), $p$ is the number of pole pairs, $\tau_{m}$ is the load torque, $B_{m}$ is the damping coefficient, $\omega_{r}$ is the rotor speed and $J_{m}$ is the moment of inertia. The basic principle in controlling a PM synchronous motor drive is based on field orientation. The flux position in the $d-q$ coordinates can be determined by the shaftposition sensor because the magnetic flux generated from the rotor permanent magnetic is fixed in relation to the rotor shaft position. In Eqs. (13-14), if $i_{d}=0$ the $d$-axis flux linkage $\lambda_{d}$ is fixed since $L_{m d}$ and $I_{f d}$ are constant for a surface-mounted PM synchronous motor, and the electromagnetic torque $\tau_{e}$ is then proportional to $i_{q}$, which is determined by closed-loop control. The rotor flux is produced in the $d$-axis only, and the current vector is generated in the $q$-axis for the field-oriented control. As the generated motor torque is linearly proportional to the $q$-axis current as the $d$-axis rotor flux is constant in Eq. (14), the maximum torque per ampere can be achieved.

With the implementation of field-oriented control, the PM synchronous motor drive system can be simplified to a control system block diagram, as shown in Fig. 2, in which

$\tau_{e}=K_{t} i_{q}^{*}$, 


$$
\begin{aligned}
& K_{t}=\frac{3}{2} P L_{m d} I_{f d}, \\
& H_{p}(s)=\frac{1}{J_{m} S+B_{m}},
\end{aligned}
$$

where $i_{q}^{*}$ is the torque current command. By substituting (16) into (15), the following applied torque can be obtained as follows:

$\tau_{m}=K_{t} i_{q}-J_{m} \& r-B_{m} \omega_{r}$ where $\tau_{m}$ is the torque applied in the direction of $\omega_{r}$, and the variables $\omega_{r}$ and $\alpha_{r}$ are the angular speed and acceleration of the disk, respectively.

According to Ref. [3], the dynamic equation of the slider-crank mechanism can be concluded to be

$$
\hat{M}(\theta) \hat{N}(\theta, \theta)=\hat{F}(\theta)
$$

Where

$$
\begin{aligned}
\hat{M}= & {\left[\left(2 m_{3}+m_{2}\right)+\frac{m_{3}}{c} r \cos \theta\right]\left(\frac{r^{3}}{c} \cos \theta \sin ^{2} \theta\right)+\left(m_{2}+m_{3}\right) r^{2} \sin ^{2} \theta+\frac{1}{3} m_{2}\left(\frac{l}{c}\right)^{2}(r \cos \theta)^{2}+\frac{1}{2} m_{1} r^{2}+J_{m} } \\
\hat{N}= & \left\{m_{2} r^{2} \sin \theta \cos \theta\left[1-\frac{l^{2}}{3 c^{2}}+\frac{r}{c} \cos \theta+\frac{(l r)^{2}}{3 c^{4}} \cos ^{2} \theta+\frac{r^{3}}{2 c^{3}} \cos \theta \sin ^{2} \theta\right]-m_{2} \frac{r^{3}}{2 c} \sin ^{3} \theta+m_{3} r^{2} \sin \theta \cos \theta\left[1-\frac{r^{2}}{c^{2}} \sin ^{2} \theta+\frac{r^{2}}{c^{2}} \cos ^{2} \theta+\frac{2 r}{c} \cos \theta\right.\right. \\
& \left.\left.+\frac{r^{4} \cos ^{2} \theta \sin ^{2} \theta}{c^{4}}+\frac{r^{3}}{c^{3}} \sin ^{2} \theta \cos \theta\right]-m_{3} \frac{r^{3}}{c} \sin ^{3} \theta\right\}+B_{m} \&_{4} \frac{1}{2} m_{2} g r \cos \theta \\
\hat{F}= & K_{t} i_{q}-\left(F_{B}+F_{E}\right) r \sin \theta\left(1+\frac{r}{c} \cos \theta\right), \\
c= & \sqrt{l^{2}-r^{2} \sin ^{2} \theta} .
\end{aligned}
$$

The system becomes an initial value problem and can be directly integrated by using the fourth order Runge-Kutta method.

\section{IDENTIFICATIONS OF THE SYSTEM}

\section{A. Particle swarm optimization}

Birds (particles) flocking optimizes a certain objective function in a PSO system. Each agent knows its best value so far (pbest) and its position. This information is analogy of personal experiences of each agent. Moreover, each agent knows the best value so far in the group (gbest) among pbests. This information is analogy of knowledge of how the other agents around them have performed. The PSO concept [5-6] consists of changing the velocity of each particle toward its pbest and gbest locations. In the PSO, each particle moves to a new position according to new velocity and the previous positions of the particle. This is compared with the best position generated by previous particles in the fitness function, and the best one is kept; so each particle accelerates in the direction of not only the local best solution but also the global best position. If a particle discovers a new probable solution, other particles will move closer to it to explore the region more completely in the process.
In general, there are three attributes, current position $x_{j}$, current velocity $v_{j}$ and past best position pbest $_{j}$, for particles in the search space to present their features. Each particle in the swarm is iteratively updated according to the aforementioned attributes. For example [5-9], the $\mathrm{jth}$ particle is represented as $\mathrm{xj}$ $=(x j, 1, x j, 2, \ldots, x j, g)$ in the g-dimensional space. The best previous position of the jth particle is recorded and represented as pbestj $=$ (pbestj,1, pbestj,2,..., pbestj,g). The index of best particle among all particles in the group is represented by the gbestg. The rate of the position change (velocity) for particle $j$ is represented as $v j=(v j, 1, v j, 2, \ldots, v j, g)$. The modified velocity and position of each particle can be calculated using the current velocity and distance from pbestj,g to gbestj,g as shown in the following formulas [9]:

$$
\begin{aligned}
& \left.v_{j, g}^{(t+1)}=w \cdot v_{j, g}^{(t)}+c_{1} \operatorname{Rand}_{(}\right) \cdot\left(\text { pbest }_{j, g}^{(t)}-x_{j, g}^{(t)}\right) \\
& +c_{2} \operatorname{Rand}^{*}() \cdot\left(\text { gbest }_{j, g}^{(t)}-x_{j, g}^{(t)}\right), \\
& x_{j, g}^{(t+1)}=x_{j, g}^{(t)}+v_{j, g}^{(t+1)} \cdot \\
& j=1,2, \Lambda, n ; g=1,2, \Lambda, m
\end{aligned}
$$

where $\mathrm{n}$ is the number of particles in a group; $\mathrm{m}$ is the number of members in a particle; $t$ is the pointer of iterations (generations); $v_{j, g}^{(t)}$ is the velocity of the particle $\mathrm{j}$ at iteration $\mathrm{t}, V_{g}^{\min } \leq v_{j, g}^{(t)} \leq V_{g}^{\max }$; $\mathrm{w}$ is the 
inertia weighting factor; $c_{1}, c_{2}$ are the acceleration constants; Rand(), Rand*() are random numbers between 0 and $1 ; x_{j, g}^{(t)}$ is the current position of particle $j$ atiteration $t$; pbestj is the pbest of particle $j$; gbestg is the gbest of the group $g$.

In the above procedures, the parameter $V_{g}^{\max }$ determine the resolution or fitness, with which regions are searched between the present position and the target position. If $V_{g}^{\max }$ is too high, particles might fly past good solutions. If $V_{g}^{\max }$ is too low, particles may not explore sufficiently beyond local solutions.

The constants $c_{1}$ and $c_{2}$ represent the weighting of the stochastic acceleration terms that pull each particle toward pbest and gbest positions. Low values allow particles to roam far from the target regions before being tugged back. On the other hand, high values result in abrupt movement toward or past target regions.

Suitable selection of inertia weighting factor tw provides a balance between global and local explorations, thus requiring less iteration on average to find a sufficiently optimal solution. As originally developed, $w$ often decreases linearly from about 0.9 to 0.4 during a run. In general, the inertia weighting factor $w$ is set according to the following equation [67]:

$$
w=w_{\max }-\frac{w_{\text {max }}-w_{\text {min }}}{\text { iter }_{\text {max }}} \times \text { iter }
$$

where iter $_{\max }$ is the maximum number of iterations (generations), and iter is the current number of iterations.

\section{B. Modified particle swarm optimization}

The main point of the MPSO differs from the PSO is to consider the "distance" in its fitness function to avoid converging to a local optimum. Assign a rank (i.e., the number place $1,2,3, \ldots$, etc.) $R E_{k}$ tothe calculated error of each new individual, $v_{k}$, $k=1, \Lambda, P S, P S$ is the population size. A combined population with $2 \times P S$ individuals is formed. Unlike previously developed statistic methods, the concept of "distance" is added to the fitness function to prevent from being trapped in a local minimum. The fitness score of the kth individual is modified by [11-12] $F_{k}=R E_{k}+\rho \times R D_{k}, k=1, \Lambda, 2 \times P S$. where $\rho$ is an adaptive decay scale, $\rho_{\max }$ is set as 0.7 and $\rho_{\min }$ is set as 0.005 in this paper. $R D_{k}$ is the rank of $D_{k}$ assigned to the kth individual, where $D_{k}$ is the distance from the individual to the current best solution vector, and is given by

$D_{k}=\left\|v_{k}-v_{\text {best }}\right\|$

where $v_{k}$ is the vector of the kth individual in the combined population, and $v_{\text {best }}$ is the current best solution vector.

An adaptive scheme is defined as [11]

$\rho_{\Delta}=R \times\left(\rho_{\text {max }}-\rho_{\text {min }}\right) / g_{\text {max }}$

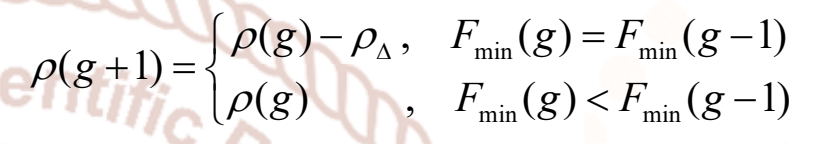

and

$\rho(g+1)=\rho_{\min } ;$ if $\rho(g)-\rho_{\Delta}<\rho_{\min }$

where $\rho_{\Delta}$ is the step size; $F_{\min }$ is the minimum value of fitness functions; $R$ is the regulating scale and is set as 1.25 in this paper, and $g_{\max }$ is the maximum allowable number of iterations.

Individuals will be ranked in ascending according to their fitness scores by a sorting algorithm. The PS individuals are transcribed along with their fitness for the next generation. If the new population does not include the current best solution, the best solution must be replaced with the last individual in the new population. In addition, a gradually decreased decay scale can satisfy a successive statistic searching process by first using the diversification (bigger $\rho$ ) to explore more regions, and then the intensification (smaller $\rho$ ) to exploit the neighborhood of an elite solution. The current best solution (point A) for a minimum fitness problem as shown in the Fig. 3 may not reach the global optimum [11-12], and there are three electable solutions exist. Generally, solutions with slightly better fitness (point C or B) prevailed, so the solution trapped into the valley prematurely. The more attractive solution(point $G$ ) is relatively far away from point A, but it nears the global optimal. To prevent prematurity, point $G$ with slightly worse fitness than $C$, it needs a higher rank to be selected. That is, a higher $R D_{k}$ is awarded toa longer $D_{k}$. 
Stopping Criteria: Stopping criteria is given in the following order:

1. Maximum allowable number of iterations reached.

2. Number of iterations reached without improving the current best solution.

Fig. 4 shows the flow chart of the proposed algorithm.

\section{Parameter identifications}

How to define the fitness function is the key point of the MPSO, since the fitness function is a figure of merit, and could be computed by using any domain knowledge. In this paper, we adopt the fitness function as follows [13-14]:

$$
F(\text { parameters })=\sum_{i=1}^{n} E_{i}^{2}
$$

$E_{i}=x^{(i)}-x^{*(i)}$

where $n$ is the total number of samples and $E_{i}$ is the calculated error of the $i_{\text {th }}$ sampling time, $x^{*(i)}$ is a solution by using the fourth-order Runge-Kutta method to solve the dynamic Equations (31) for the PM synchronous servomotor drive coupled with a slider-crank mechanism with the parameters identified from these two methods, and $x^{(i)}$ is the displacement measured experimentally at the ${ }^{i}$ th sampling time.

\section{SIMULATIONS AND EXPERIMENTAL RESULTS}

A block diagram of the computer control system for the PM synchronous servomotor drive coupled with a slider-crank mechanism is shown in Fig. 5(a) and the experimental equipments of a slider-crank mechanism of computer control system is shown in Fig. 5(b). The control algorithm is implemented using a Pentium computer and the control software is LABVIEW. The PM synchronous servomotor is implemented by MITSUBISHI HC-KFS43 series. The specifications are shown as follows: Rated output 400 (W), Rated torque $1.3(\mathrm{Nm})$, Rated rotation speed $3000(\mathrm{rpm})$ and Rated current 2.3 (A). The servo is implemented by MITSUBISHI MR-J2S-40A1. The control system is Sine-wave PWM control, which is a current control system. In order to measure the angle and angular speed of the disk and the position and velocity of the slider B, the interface of the device is implemented by motion control card PCI-7342. It can measure the angle of the disk and the position of slider B at the same time.
The main parameters of a slider-crank mechanism and servomotor used in the numerical simulations and the experiments are as follows

$m_{1}=0.232 \mathrm{~kg}, m_{2}=0.332 \mathrm{~kg}$,

$m_{3}=0.600 \mathrm{~kg}, r=0.030 \mathrm{~m}$,

$l=0.217 \mathrm{~m}, F_{B}=0.100 \mathrm{~N}$,

$F_{E}=0.000 \mathrm{~N}, i_{q}=0.400 \mathrm{~A}$,

$K_{t}=0.5652 \mathrm{Nm} / \mathrm{A}, J_{m}=6.700 \cdot 10^{-5} \mathrm{Nms}^{2}$,

$B_{m}=1.430 \cdot 10^{-2} \mathrm{Nms} / \mathrm{rad}$.

The physical model of the slider-crank mechanism driven by a servomotor. In the parameter identification, we utilize the MPSO and PSO methods to identify the 5 parameters $m_{1} 、 m_{2} 、 m_{3} 、 r$ and $l$ simultaneously, and the fitness function is described as Eq. (20). The identified results are given in Table 1.

Figure 6 shows the displacement errors of the slidercrank mechanism by both the PSO and MPSO methods. Furthermore, their displacement errors are all about $\pm 0.2^{\mu \mathrm{m}}$. Figure 7 shows the convergence characteristics in PSO and MPSO methods of the slider-crank mechanism system. It is seen from Fig. 7 that the proposed MPSO method is superior to the PSO method.

\section{CONCLUSION}

The dynamic formulations of a slider-crank mechanism driven by a field-oriented PM synchronous motor have been successfully formulated with only one independent variable. Furthermore, the main objective of this study is to utilize PSO and MPSO methods to identify a slider-crank mechanism driven by a servomotor. According to the comparisons between identified results and displacement errors, it is found that MPSO method has the best matching with the experimental results.

It is concluded that the implementations of MPSO are different from the PSO in five aspects. Firstly, its fitness function considers the distance to avoid converging to a local optimum. Secondly, for the MPSO, vectors with good enough fitness scores would be used as candidates to create new solutions. Thirdly, it has the advantage of the MPSO to conquer various constraints without using the fitness function with penalties, and can perform better. Fourthly, the solution is coded with a decimal representation, and saves computer memory. Finely, the gradually 
decaying parameters can satisfy a successive statistic searching process by first using the diversification (bigger parameters) to reserve the larger attractive region. Then, the intensification (smaller parameters) used to search the small neighborhood of an elite solution.

\section{REFERENCES}

1. Viscomi, B. V., and Arye, R. S., "Nonlinear Dynamic Response of Elastic Slider-Crank Mechanism" ASME J. of Eng. for Industry, Vol. 93, pp. 251-262, 1971.

2. Fung, R. F., "Dynamic Response of the Flexible Connecting Rod of a Slider-Crank Mechanism with Time-Dependent Boundary Effect" Computer \& Structure, 63, No. 1, pp. 79-90, 1997.

3. Fung, R. F., Lin, F. J., Huang, J. S. and Wang, Y. C., "Application of Sliding Mode Control with a Low-pass Filter to the Constantly Rotating SliderCrank Mechanism" The Japan Society of Mechanical Engineers, C, 40, No. 4, pp 717-722, 1997.

4. Lin, F. J., Fung, R. F., and Lin, Y. S., "Adaptive Control of Slider-Crank Mechanism Motion: Simulation and Experiments", International Journal of System Science, 28, No. 12, pp. 1227 1238, 1997.

5. Kennedy, J., Eberhart, R. C., "Particle Swarm Optimization", Proceedings of the IEEE International Joint Conference on Neural Networks, IEEE Press, pp. 1942-1948, 1995.

6. Eberhart, R. C., Kennedy, J., "A New Optimizer Using Particle Swarm Theory", Proceedings of the Sixth International Symposium on Micro machine and Human Science, Nagoya, Japan, pp.39-43, 1995.

7. Kennedy, J., "The Particle Swarm: Social Adaptation of Knowledge", Proceedings of the IEEE International Conference on Evolutionary Computation, Indianapolis, USA, pp. 303-308, 1997.

8. Naka, S., Genji, T., Yura, T., Fukuyama, Y., "A Hybrid Particle Swarm Optimization for Distribution State Estimation", IEEE Transactions on Power systems Vol. 18, No.1, pp.60-68, 2003.

9. Shi, Y., Eberhart, R. C., "Empirical Study of Particle Swarm Optimization", Proceedings of the IEEE Congress on Evolutionary Computation, IEEE Press, pp.1945-1950, 1999.
10. Krause, P. C., Analysis of Electric Machinery (New York: McGraw-Hill), 1986.

11. Lin, W. M., Cheng, F. S., and Tsay, M. T., "Nonconvex Economic Dispatch by Integrated Artificial Intelligence", IEEE Transactions on Power systems, Vol. 16, No. 2, pp. 307-311, 2001.

12. Lin, W. M., Cheng, F. S., and Tsay, M. T., "An Improved Tabu Search for Economic Dispatch With Multiple Minima", IEEE Transactions on Power systems, Vol. 17, No. 1, pp. 108-112, 2002.

13. Zhang, X., Huang, Y., Liu, J., Wang, X., and Gao, F., "A Method Identifying the Parameters of Bouc-Wen Hysteretic Nonlinear Model Based on Genetic Algorithm", Intelligent Processing Systems, pp. 602-605, 1997.

14. Ha, J. L., Kung, Y. S., Fung, R. F., and Hsien, S. C., "A Comparison of Fitness Functions for the Identification of Piezoelectric Hysteretic Actuator Based on the Real-Coded Genetic Algorithm", Sensors and Actuators, Vol.132, pp.643-650, 2006.

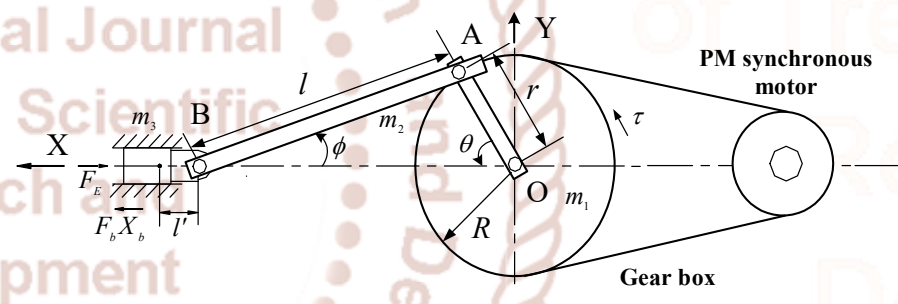

Fig. 1(a) The physical model of a slider-crank mechanism.

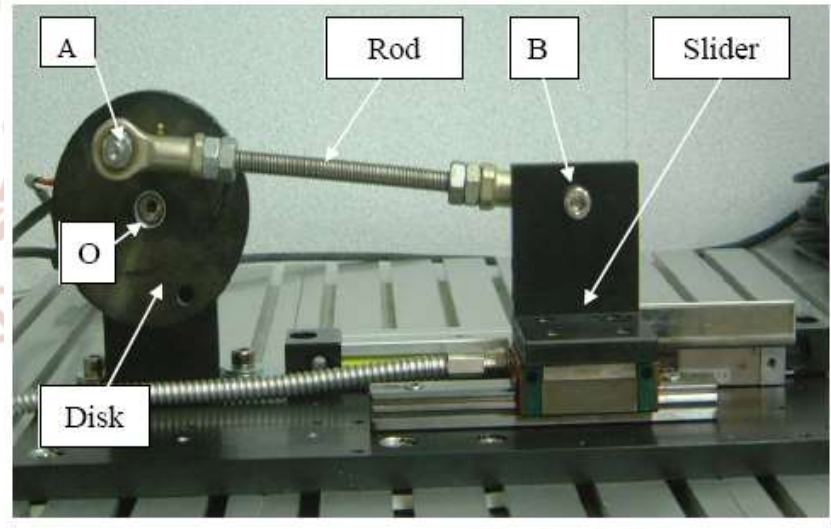

Fig. 1(b) The experimental equipments of a slidercrank mechanism

Fig. 1 The slider-crank mechanism

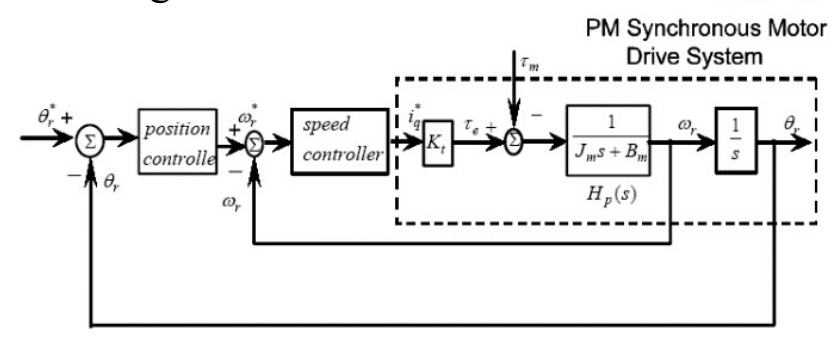


International Journal of Trend in Scientific Research and Development (IJTSRD) ISSN: 2456-6470

Fig.2 Simplified control block diagram

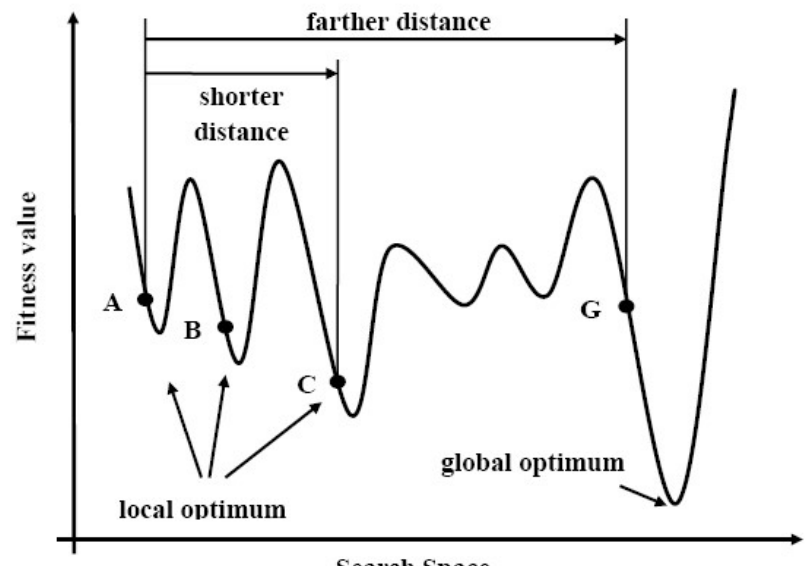

Fig.3 The concept of distances.

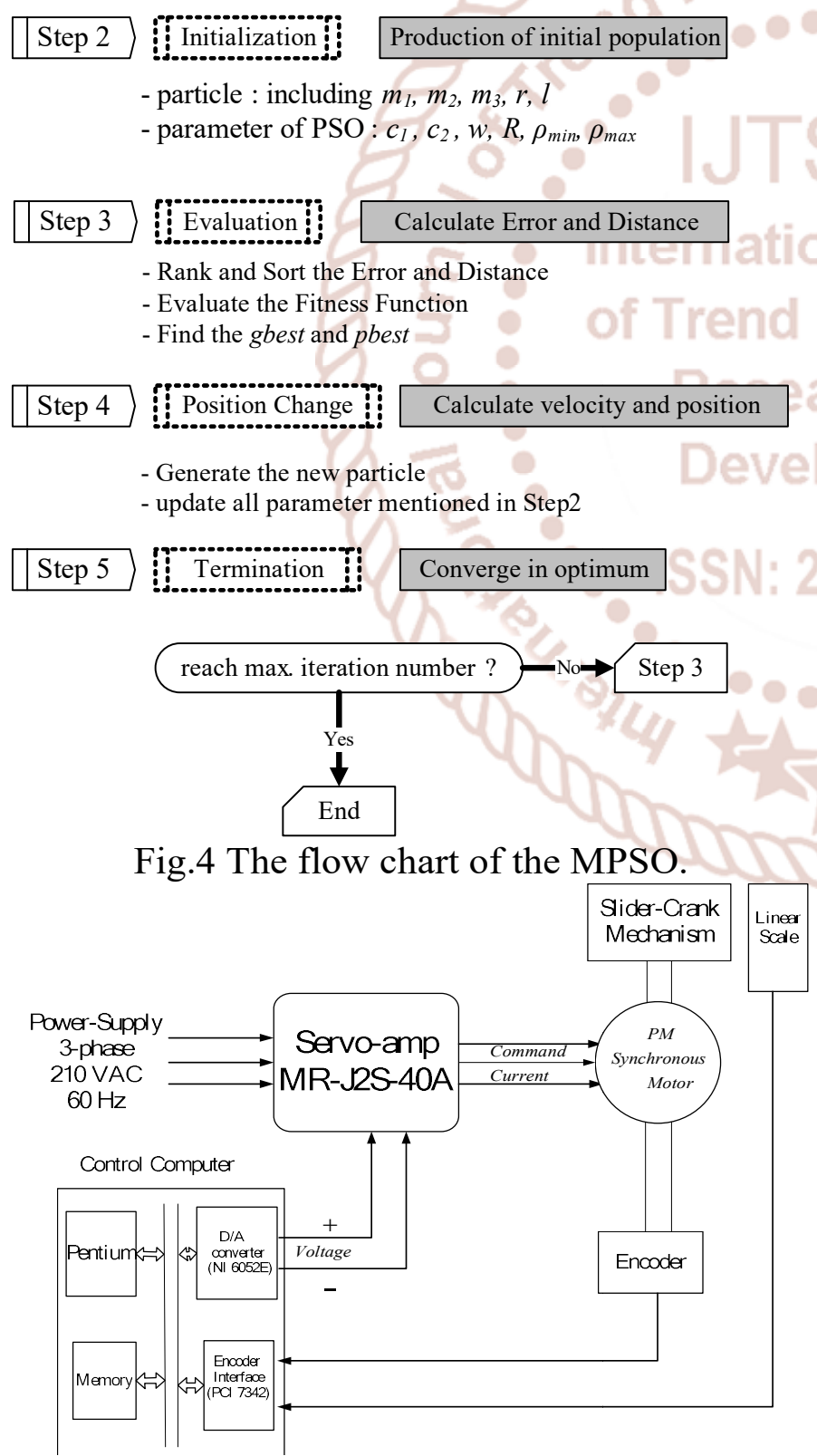

(a)

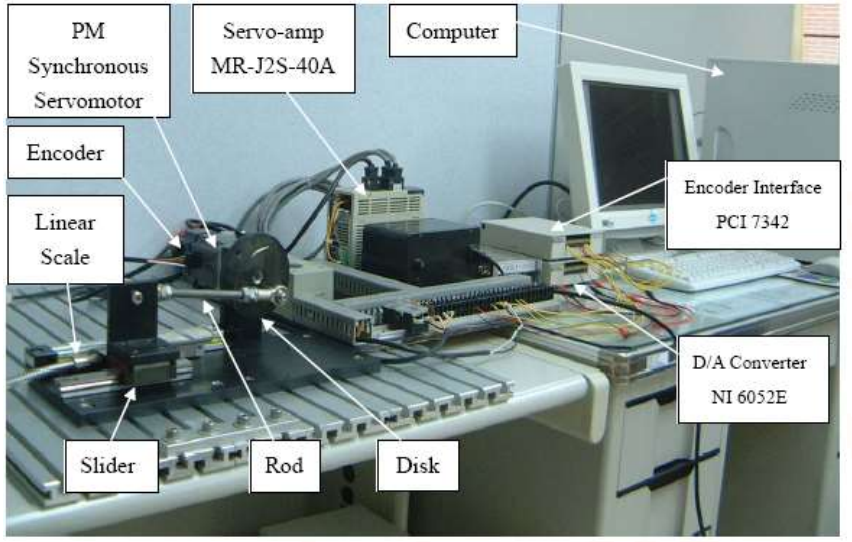

(b)

Fig.5 The experimental setup. (a) Computer control system block diagram; (b) The experimental equipments of a slider-crank mechanism of Computer control system.

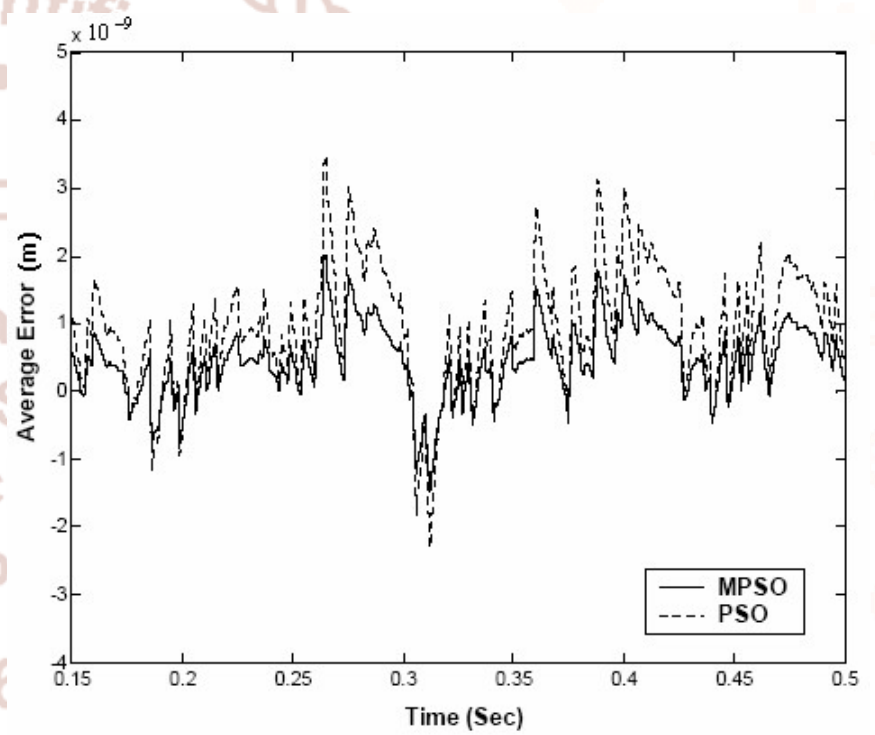

Fig. 6 The comparisons in displacement errors between the PSO and MPSO methods.

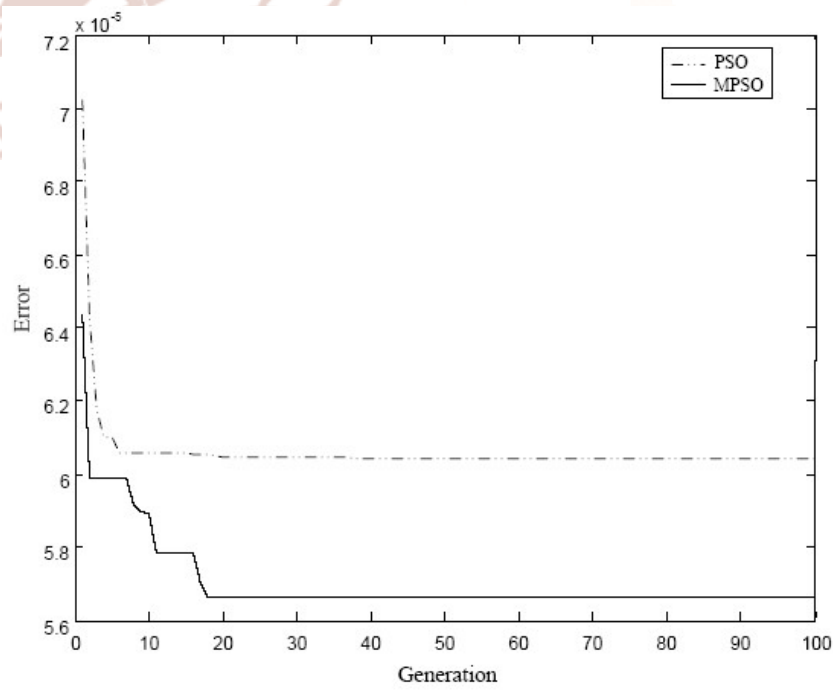

Fig. 7 Comparison of convergence characteristics in PSO and MPSO methods of the slider-crank mechanism system. 
International Journal of Trend in Scientific Research and Development (IJTSRD) ISSN: 2456-6470

Table 1. The identified parameters of the numerical simulations.

\begin{tabular}{|c|c|c|c|c|c|}
\hline Parameter & $m_{1}(\mathrm{~kg})$ & $m_{2}(\mathrm{~kg})$ & $m_{3}(\mathrm{~kg})$ & $r(\mathrm{~m})$ & $l(\mathrm{~m})$ \\
\hline Feasible domain & $0.000 \sim 1.000$ & $0.000 \sim 1.000$ & $0.000 \sim 1.000$ & $0.000 \sim 0.100$ & $0.000 \sim 1.000$ \\
\hline The actual value & 0.232 & 0.332 & 0.600 & 0.030 & 0.217 \\
\hline $\begin{array}{c}\text { The identified value of the } \\
\text { PSO method }\end{array}$ & 0.311 & 0.304 & 0.752 & 0.024 & 0.325 \\
\hline $\begin{array}{c}\text { The identified value of the } \\
\text { MPSO method }\end{array}$ & 0.234 & 0.331 & 0.603 & 0.030 & 0.216 \\
\hline
\end{tabular}

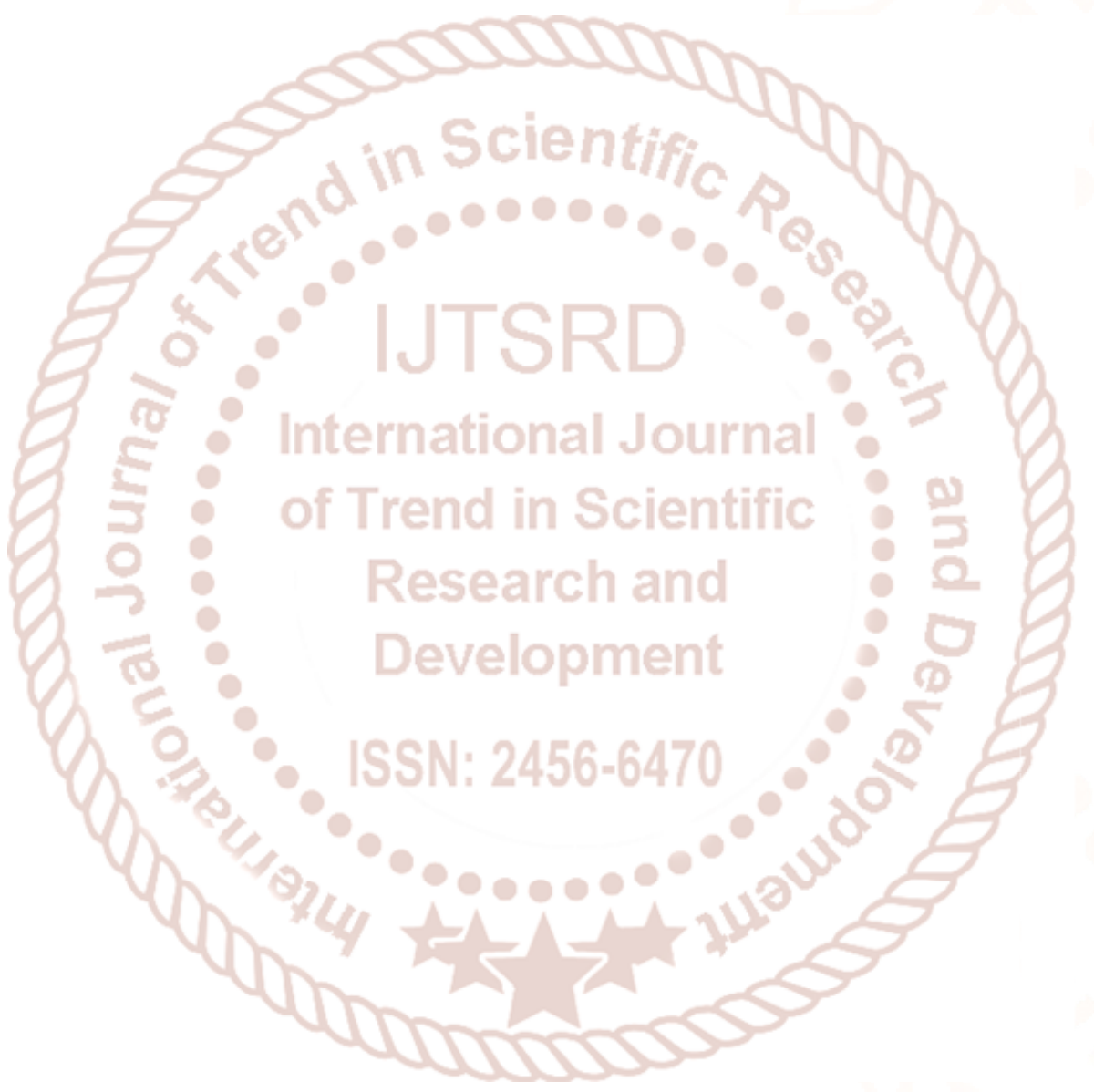

